Modeling anti-KLH ELISA data using two-stage and mixed effects models in support of immunotoxicological studies

Peer-reviewed author version

SHKEDY, Ziv; STRAETEMANS, Roel; MOLENBERGHS, Geert; Desmidt, Miek; Vinken, Petra; Goeminne, Nick; Coussement, Werner; Van den Poel, Bob \& BIJNENS, Luc (2005) Modeling anti-KLH ELISA data using two-stage and mixed effects models in support of immunotoxicological studies. In: JOURNAL OF BIOPHARMACEUTICAL STATISTICS, 15(2). p. 205-223.

DOI: 10.1081/BIP-200048815

Handle: http://hdl.handle.net/1942/2037 


\title{
Modeling Anti-KLH ELISA Data Using Two-Stage and Mixed Effects Models in Support of Immunotoxicological Studies
}

\author{
Ziv Shkedy, Roel Straetemans, Geert Molenberghs \\ Limburgs Universitair Centrum, Center for Statistics, Biostatistics, Universitaire Campus, \\ Miek Desmidt, Petra Vinken, Nick Goeminne, Werner Coussement, Bob Van Den Poel \\ and \\ Luc Bijnens \\ J\&JPRD, Beerse, Belgium
}

SUMMARY

During preclinical drug development the immune system is specifically evaluated after prolonged treatment with drug candidates, since the immune system may be an important target system. The response of antibodies against a T-cell dependent antigen is recommenced by the FDA and EMEA for the evaluation of immunosuppression/enhancement. For that reason we developed a semi-quantitative enzyme-linked immunosorbent assay to measure antibodies against keyhole limpet hemocyanin. The analysis of this kind of data is at this moment not yet fully explored. In this paper we describe two approaches for modeling immunotoxic data using nonlinear models. The first is a two stage model in which we fit an individual nonlinear model for each animal in the first stage and the second stage consists of testing possible treatment effects using the individual maximum likelihood estimates obtained in the first stage. In the second approach the inference about treatment effects is based on a nonlinear mixed model which account for heterogeneity between animals. In both approaches we use a three parameter logistic model for the mean structure.

Keywords:

EMEA; ELISA; FDA, Immunotoxicological studies; KLH; Nonlinear mixed effects model; Two stage model; Variance components; 


\section{Introduction}

In humans and in animals the immune system may be an important target system, exhibiting a unique susceptibility to drugs and other xenobiotics. As a result, the immune system is specifically evaluated after prolonged treatment with drug candidates during preclinical drug development. In recently issued guidance of regulatory agencies in Europe (1), the United States (2) and Japan (3) the antibody response against a T-cell dependent antigen is recommended to evaluate immunosuppression or enhancement. A semi-quantitative enzyme-linked immunosorbent assay (ELISA) to measure antibodies present in blood after immunisation of rats with keyhole limpet hemocyanin $(\mathrm{KLH})$, a T-cell dependent antigen was developed and validated according to ????guideline. The method is considered semi-quantitative because it does not include a quantified reference standard. The anti-KLH antibodies are measured in series of dilutions of samples and expressed as optical density values. These optical densities are a measure of the specific antibody level in a sample. The optical density is high when the antibody levels are high as a result of a strong immune reaction. The relationship between the serial dilutions and the optical densities is typically sigmoid. Many classical methods used to analyse these data concentrate on the OD50 alone (reference to book Nick). That way differences between curves may not be seen if they originate for example from differences in the maximums. In this paper we describe two approaches that both provide estimates of three different shape parameters of the sigmoidal curves using nonlinear models. Parameters of primary interest are : (1) the maximum optical density, (2) the slope and (3) the dilution value corresponding to $50 \%$ of the maximum (OD50). The first model we consider is a two stage approach in which we fit an individual nonlinear model for each animal in the first stage. The second stage consists of testing possible treatment effects using the individual maximum likelihood estimates obtained in the first stage. In the second method the inference

about treatment effect is based on a nonlinear mixed model which account for heterogeneity between animals. In both approaches we use a three parameter logistic model for the mean structure. To our knowledge no comprehensive statistical methods are available for this kind of ELISA's applied in immunotoxicology.

This paper is orgenzed as follows. The data are introduced in Section 2 while Section 3 
described the two modeling approaches: the two-stage apparoch and the mixed modeliong approach. The proposed methods apply to the data in Section 4. In Section 5 we focus on comutetional issues where we discuss the avilable software (SAS/SPlus).

\section{The immunotoxic data}

Thirty adult Sprague-Dawley rats (Charles River, Sulzfeld, Germany) were allocated to 2 treatment groups 1 and 2 (5 male and 10 female rats per group). Rats were administered a single intraperitoneal injection on Day 0 with 200 (group 1) or 800 g KLH (group 2) per rat. A blood sample was taken on Day 14 for anti-KLH antibody determination by ELISA. Briefly, microtiter plates were coated with ????KLH for 1 day at $4 \mathrm{C}$. Plates were then washed and blocked with ?????? overnight at 4 C. Serum samples were diluted 1:15 in PBS containing 0.05\% Tween 20 (Sigma) and a single 3-fold serial dilution was performed. Microtiter plates were incubated for ??? at ??? C and then washed. Secondary antibody anti-rat IgM conjugated to horseradish peroxydase was added to each well. Plates were incubated and washed before addition of ABTS (Sigma) and enzyme substrate. Plates were incubated and the optical densities were measured after ??? minutes at $405 \mathrm{~nm}$ on a plate reader (Milenia Kinetic Analyser, Molecular Devices). A serial dilution of a pooled positive control serum was added.

Figure 2 shows the animal profiles of the response values versus dilution. Both figures

reveal three patterns: (1) a nonlinear relationship between the response and dilution (and log dilution), (2) considerable amount of variability between animals and (3) decreasing variability with dilution. For the analysis presented in this report we use log dilution as predictor.

FIGURE 1 and FIGURE 2 ABOUT HERE 


\section{$3 \quad$ Modeling strategy}

The model which was used to describe the relationship between the response and log dilution is a three parameters logistic model of the form

$$
y_{i j}=\frac{\theta_{1 i}}{1+\left(\frac{C_{i j}}{\theta_{2 i}}\right)^{\theta_{3 i}}}+\varepsilon_{i j}, \quad i=1, \ldots, n, j=1, \ldots, J .
$$

Here, $y_{i j}$ is the response of animal $i$ at dilution $j, C_{i j}$ is the $\log$ (dilution) and $\varepsilon_{i j}$ is the measurement error which is assumed to be normally distributed with mean zero and covariance matrix $\boldsymbol{\Sigma}$. The nonlinear model in (1) can be written as

$$
y_{i j}=f\left(\boldsymbol{\theta}_{i}, C_{i j}\right)+\varepsilon_{i j}
$$

where $f$ is the nonlinear function in (1) and $\boldsymbol{\theta}_{i}=\left(\theta_{1 i}, \theta_{2 i}, \theta_{3 i}\right)$ is an animal-specific parameter vector to be estimated. The parameter $\theta_{1 i}$ is the maximum effect which is achieved as $C_{i j} \longrightarrow 0, \theta_{2 i}$ is the dilution value at which the response is $\theta_{1 i} / 2$ and $\theta_{3 i}$ is the slope.

\subsection{Two Stage Approach}

\subsubsection{Formulation of The Two Stage Model}

In the first approach a two stage model is considered. The first stage consists of fitting the nonlinear model in (1) for each animal separately, resulting in animal-specific ML estimates $\hat{\boldsymbol{\theta}}_{i}=\left(\hat{\theta}_{1 i}, \hat{\theta}_{2 i}, \hat{\theta}_{3 i}\right)$. In the second stage of the model we specify the joint distribution of $\hat{\boldsymbol{\theta}}$

$$
\left(\begin{array}{c}
\hat{\theta}_{1 i} \\
\hat{\theta}_{2 i} \\
\hat{\theta}_{3 i}
\end{array}\right) \sim \mathrm{N}\left(\left(\begin{array}{c}
\theta_{1 i} \\
\theta_{2 i} \\
\theta_{3 i}
\end{array}\right), \mathbf{A}\right), \quad \mathbf{A}=\left(\begin{array}{lll}
a_{11} & a_{12} & a_{13} \\
a_{21} & a_{22} & a_{23} \\
a_{31} & a_{32} & a_{33}
\end{array}\right), \quad i=1, \ldots, n .
$$

Here, $\left(\theta_{1 i}, \theta_{2 i}, \theta_{3 i}\right)$ are the true animal specific parameters and $\mathbf{A}$ is the covariance matrix which accounts for possible correlation among the ML estimates. It is further assumed that the true animal specific parameters follow the following structural model

$$
\left(\begin{array}{c}
\theta_{1 i} \\
\theta_{2 i} \\
\theta_{3 i}
\end{array}\right) \sim \mathrm{N}\left(\left(\begin{array}{c}
\beta_{1}+\gamma_{1} I_{i} \\
\beta_{2}+\gamma_{2} I_{i} \\
\beta_{2}+\gamma_{2} I_{i}
\end{array}\right), \mathbf{B}\right), \quad \mathbf{B}=\left(\begin{array}{lll}
b_{11} & b_{12} & b_{13} \\
b_{21} & b_{22} & b_{23} \\
b_{31} & b_{32} & b_{33}
\end{array}\right), i=1, \ldots, n .
$$


Here, $I_{i}$ is an indicator variable which takes the value of 1 if the $i$ 'th animal belongs to the first treatment group and zero otherwise. Combining the models in (3) and (4) leads to the marginal model for the ML estimates

$$
\left(\begin{array}{c}
\hat{\theta}_{1 i} \\
\hat{\theta}_{2 i} \\
\hat{\theta}_{3 i}
\end{array}\right) \sim \mathrm{N}\left(\left(\begin{array}{c}
\beta_{1}+\gamma_{1} I_{i} \\
\beta_{2}+\gamma_{2} I_{i} \\
\beta_{3}+\gamma_{3} I_{i}
\end{array}\right), \mathbf{D}\right)
$$

\subsubsection{Testing For Treatment Effects}

In the case both $\mathbf{A}$ and $\mathbf{B}$ are diagonal matrices, univariate one-way ANOVA can be used to test treatment effects. Formally, for each one of the parameters, we wish to test the hypotheses

$$
\begin{aligned}
& H_{0, i}: \gamma_{i}=0, \\
& H_{1, i}: \gamma_{i} \neq 0 .
\end{aligned}
$$

Taking into account the multivariate distribution of $\boldsymbol{\theta}$ in (5), one can test the null hypothesis $H_{0}: \gamma=0$ against the alternative $H_{1}: \gamma \neq 0$ using multivariate ANOVA (for example, using Hotelling's $T^{2}$ or Wilk's Lambda).

An alternative to multivariate ANOVA for testing treatment effects is to fit the model in (5) and test the hypotheses

$$
\begin{aligned}
& H_{0}: \gamma_{1}=\gamma_{2}=\gamma_{3}=0 \\
& H_{1}: \text { at least one } \gamma_{i} \text { is not equal to zero. }
\end{aligned}
$$

Note that Model (5) can be expressed as

$$
\hat{\boldsymbol{\theta}}_{i} \sim \mathrm{N}\left(\boldsymbol{X}_{i} \boldsymbol{\beta}, \mathbf{D}\right)
$$

with

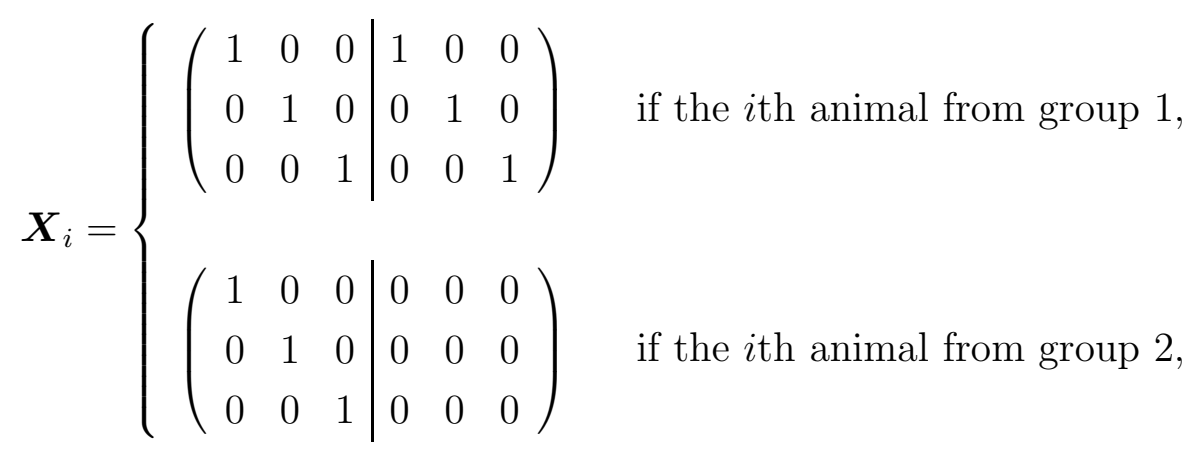


and the parameter vector $\boldsymbol{\beta}=\left(\beta_{1}, \beta_{2}, \beta_{3}, \gamma_{1}, \gamma_{2}, \gamma_{3}\right)$. Hence,

$$
\left(\begin{array}{c}
\theta_{1} \\
\theta_{2} \\
\theta_{3}
\end{array}\right)=\left\{\begin{array}{l}
\beta_{1}+\gamma_{1}, \\
\beta_{2}+\gamma_{2}, \\
\beta_{3}+\gamma_{3}, \\
\beta_{1}, \\
\beta_{2}, \\
\beta_{3} .
\end{array} \quad \text { animal from group } 2\right.
$$

In order to test treatment effects one can use the likelihood ratio test in order to compare a reduced model, which does not include treatment effects, and the model in (5).

\subsection{Nonlinear Mixed Effects Model}

\subsubsection{Formulation of The Mixed Model}

Nonlinear mixed effects models combines the two stages in (1) and (3) into one model. For the $i$ th animal it is assumed

$$
\mathbf{y}_{i j}=f\left(\boldsymbol{\theta}_{i}, C_{i j}\right)+\varepsilon_{i j}
$$

where $f$ is the nonlinear function in $(1), \boldsymbol{\theta}_{i}$ is a animal specific parameter vector and $\boldsymbol{\varepsilon}_{i}=$ $\left(\varepsilon_{i 1}, \ldots, \varepsilon_{i n}\right)$ is a random error term. The animal-specific parameter vector is modelled as

$$
\boldsymbol{\theta}_{i}=\boldsymbol{X}_{i} \boldsymbol{\beta}+\boldsymbol{Z}_{i} \boldsymbol{b}_{i}
$$

Here, $\boldsymbol{\beta}$ is a fixed parameters vector, $\boldsymbol{b}_{i}$ is a animal-specific random effects vector and $\boldsymbol{X}_{i}$ and $\boldsymbol{Z}_{i}$ are known design matrices for the fixed effects $\boldsymbol{\beta}$ and the random effects $\boldsymbol{b}_{i}$, respectively:

$$
\boldsymbol{X}_{i}=\boldsymbol{Z}_{i}=\left(\begin{array}{ccc}
1 & 0 & 0 \\
0 & 1 & 0 \\
0 & 0 & 1
\end{array}\right) \quad \boldsymbol{\beta}=\left(\begin{array}{c}
\beta_{1} \\
\beta_{2} \\
\beta_{3}
\end{array}\right) \quad \text { and } \quad \boldsymbol{b}_{i}=\left(\begin{array}{c}
b_{i 1} \\
b_{i 2} \\
b_{i 3}
\end{array}\right)
$$

It follows from (10) that the animal-specific parameter vector can be expressed as

$$
\left(\begin{array}{c}
\theta_{1 i} \\
\theta_{2 i} \\
\theta_{3 i}
\end{array}\right)=\left(\begin{array}{c}
\beta_{1}+b_{i 1} \\
\beta_{2}+b_{i 2} \\
\beta_{3}+b_{i 3}
\end{array}\right)
$$


The random effects $\boldsymbol{b}_{i}$ and the error terms $\boldsymbol{\varepsilon}_{i}$ are assumed to be normally distributed as $\boldsymbol{b}_{i} \sim \mathrm{N}(0, \mathbf{D})$ and $\boldsymbol{\varepsilon}_{i} \sim \mathrm{N}(0, \boldsymbol{\Sigma})$. The structure of the design matrices $\mathbf{D}$ and $\boldsymbol{\Sigma}$ will be discussed in the Section 3.

\subsubsection{Testing for Treatment Effects}

To test for possible treatment effects, one needs to modify the configuration of the design matrix for the fixed effect in (11) to the configuration given in (8) with the parameter vector

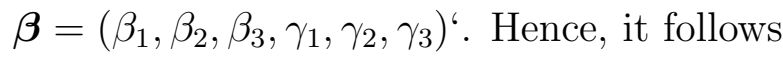

$$
\theta_{k i}= \begin{cases}\beta_{k}+\gamma_{k}+b_{i k} & \text { if the } i \text { th animal from group } 1 \\ \beta_{k}+b_{i k} & \text { if the } i \text { th animal from group } 2\end{cases}
$$

\section{Application to the Data}

In this section we applied the both methods to the data. We first discuss the results obtained from the two stage model discussed in Section 2.1 and then present the results obtained using the mixed effects model discussed in Section 2.2. A sequence of mixed effects models was fitted in order to find the structure for $\boldsymbol{\Sigma}$ and $\mathbf{D}$ which have the best fit to the data.

\subsection{Two Stage Model}

The nonlinear model (1) was fitted for each animal separately. Figures 3 and 4 show the individual models. Figure 5 shows the animal-specific ML estimates (and 95\% C.I). The first 15 lines in this plot show the ML estimates in the first treatment group while the next 15 lines show the ML estimates obtained in the second treatment group. A remarkable between animal variability is observed among the ML estimates but is seems that there is no difference between the treatment groups. 


\subsubsection{Univariate and multivariate ANOVA}

Results obtained from the univariate and multivariate ANOVA are shown in Table 1. The null hypothesis in (6) of no treatment effects cannot be rejected for all of the univariate cases. The Wilks Lambda statistics is equal to $0.986(\mathrm{P}=947)$, i.e. the null hypothesis of no treatment effects cannot be rejected.

\section{TABLE 1 ABOUT HERE}

\subsubsection{Marginal Distribution for $\theta$}

The marginal model $\hat{\boldsymbol{\theta}}_{i} \sim \mathrm{N}\left(\boldsymbol{X}_{i} \boldsymbol{\beta}, D\right)$ was fitted using the Splus function gls(). Parameter estimates obtained from the marginal model are shown in Table 2. The parameter estimates for the treatment effects obtained from the second-stage model, are $\hat{\gamma}_{1}=$ 0.000075 (s.e. 0.219 ), $\hat{\gamma}_{2}=-0.091$ (s.e. 0.291 ) and $\hat{\gamma}_{3}=-0.379$ (s.e. 0.756 ) found to be nonsignificant. To test for treatment effects we used the likelihood ratio test in order between a model which includes treatment effects and a model which does not include treatment effects (i.e. $\left.E(\hat{\boldsymbol{\theta}})=\left(\beta_{1}, \beta_{2}, \beta_{3}\right)\right)$. The likelihood ratio statistics is equal to 0.414 on 3 degrees of freedom $(\mathrm{P}$-value $=0.937)$ indicates that the null hypothesis of no treatment effects cannot be rejected.

FIGURE ??,FIGURE ??,FIGURE 3,FIGURE 4,FIGURE 5 and TABLE 2 ABOUT HERE

\subsection{Nonlinear Mixed Model}

\subsubsection{Model 1: Unstructured Covariance Matrix for $b_{i}$ and Uncorrelated Resid- ual Errors}

The nonlinear mixed model specified in (10)-(??) was fitted with covariance structure given by $\boldsymbol{b}_{i} \sim \mathrm{N}\left(0, \mathbf{D}_{1}\right)$ and $\boldsymbol{\varepsilon}_{i} \sim \mathrm{N}\left(0, \sigma^{2} \boldsymbol{I}\right)$. In this stage, the covariance matrix for the random 
effects is assumed to be unstructured, i.e.,

$$
\mathbf{D}_{1}=\left(\begin{array}{lll}
d_{11} & d_{12} & d_{13} \\
d_{21} & d_{22} & d_{32} \\
d_{31} & d_{23} & d_{33}
\end{array}\right)
$$

Note that we do not, at this stage, include treatment effects into the model. Parameter

estimates for $\boldsymbol{\theta}$ are equal to $\hat{\beta}_{1}=1.751(0.107), \hat{\beta}_{2}=5.513(0.129), \hat{\beta}_{3}=8.002(0.397)$ and they are comparable to those which were obtained from the marginal model of the two stage model. The residual variance was estimated to be 0.016 .

\subsubsection{Model 2: Unstructured Covariance Matrix for $b_{i}$ and Power Variance Function for the Residual Errors}

Figure 6 shows the standardized residuals versus $C_{i j}$. Clearly the variability decreases with $C_{i j}$. We therefore modified the structure of $\boldsymbol{\Sigma}$ and included a power function $\Sigma=\sigma^{2} \times$ $\left|C_{i j}\right|^{2 \delta} \boldsymbol{I}$. For $\delta<0$ the variance decreases with $C_{i j}$.

Parameter estimates are equal to $\hat{\beta}_{1}=1.872$ (s.e. 0.106$), \hat{\beta}_{2}=5.336$ (s.e. 0.112 ), $\hat{\beta}_{3}=$ 6.944 (s.e. 0.142). The estimated value for the power is -3.56 and $\hat{\boldsymbol{\Sigma}}=16.59^{2}\left|C_{i j}\right|^{2(-3.56)} \boldsymbol{I}$. Figure 7 (left panel) shows the variance functions for both models. Note that compared with Model 1, Model 2 predicted decreasing variability with dilution. The AIC for Model 1 is equal to -67.87 and it decreases substantially for model $2(-488.55)$ indicating that the later model is preferable.

FIGURE 6 and FIGURE 7 ABOUT HERE

\subsubsection{Model 3: Block Diagonal Covariance Matrix for $b_{i}$ and Power Variance Function for the Residual Error}

Figure 8 shows the scatterplot matrix for the estimated random effects of Model 2. Figure 8 suggests that the covariance matrix $\mathbf{D}_{1}$ might be overparameterized. The estimated 
unstructured covariance matrix is given by (off diagonal elements are correlations).

$$
\hat{\mathbf{D}}_{1}=\left(\begin{array}{rrr}
0.303 & (0.372) & (0.314) \\
& 0.339 & (0.856) \\
& & 0.461
\end{array}\right) \text {. }
$$

The correlations between $\boldsymbol{b}_{1}$ to $\boldsymbol{b}_{2}$ and $\boldsymbol{b}_{3}$ are (0.372 and 0.314, respectively) relatively small when compared with the correlation between $\boldsymbol{b}_{2}$ and $\boldsymbol{b}_{3}(0.856)$. Therefore, we define a block diagonal covariance matrix for the random effects,

$$
\mathbf{D}_{2}=\left(\begin{array}{lll}
d_{11} & 0 & 0 \\
0 & d_{22} & d_{32} \\
0 & d_{23} & d_{33}
\end{array}\right)
$$

\section{FIGURE 8 ABOUT HERE}

Note that models 2 and 3 have the same mean structure and variance function for the error and differ from each other in the covariance matrix $\mathbf{D}$. Thus, we wish to test the hypotheses

$$
\begin{array}{ll}
H_{0}: d_{12}=0 \text { and } d_{13}=0 & \text { (covariance structure of model 3) } \\
H_{1}: d_{12} \neq 0 \text { and } d_{13} \neq 0 & \text { (covariance structure of model 2) }
\end{array}
$$

Parameter estimates for Models 2 and 3 are shown in Table 3. According to AIC and BIC criteria Model 3 is preferable. Formally, the likelihood ratio statistics (equal to 3.873) should be compared to a critical value from $\chi_{2}^{2}$ which equals to 5.99 (for significance level of 0.05). Therefore, the null hypothesis cannot be rejected. The estimated variance components are (off diagonal elements are correlations)

$$
\hat{\mathbf{D}}_{2}=\left(\begin{array}{lll}
0.319 & 0 & 0 \\
0 & 0.346 & (0.840) \\
0 & & 0.458
\end{array}\right) \text { and } \hat{\boldsymbol{\Sigma}}=15.75^{2}\left|C_{i j}\right|^{2(-3.53)} \boldsymbol{I} .
$$

\subsubsection{Model 4: Testing for Treatment Effects}

To test for possible treatment effects, we keep the covariance structure of Model 3 and replace the design matrix for the fixed effects to the one given in (??). The parameter estimates for the treatment effects (shown in the third column of Table 3) are all found to be 
nonsignificant. The likelihood ratio statistics, comparing between Models 3 and 2, is equal to 1.026 on 3 degrees of freedom $(\mathrm{p}$-value $=0.7949)$ indicating that the treatment effects are not needed in the model.

\subsection{Interpretation and Diagnostics for Model 3}

The estimated population averaged model is shown in Figure 9, parameters estimates are

given in Table 4. The fixed effect $\hat{\beta}_{1}=1.875$ is the maximum effect which is achieved when $C_{i j} \rightarrow 0$. $\hat{\beta}_{2}=5.369$ is the value of $\log$ dilution for which the response is $\hat{\beta}_{1} / 2$, this correspond to a dilution of 214.77. For the two stage model $\hat{\beta}_{2}=5.45$ which corresponds to dilution of 232.96. Population models for model 3 and the two stage model are shown in Figure 9 .

\section{FIGURE 9, AND TABLE 4 ABOUT HERE}

For Model 4 (which include treatment effects) the standard error for the parameter estimates obtained from the mixed-effects model are smaller than those which are obtained from the two-stage model. Figure ?? shows the observed and fitted values for both fixed effects and animal specific models. In Figures 10 (treatment group 1) and 11 (treatment group 2) a plot of the observed versus predicted values are shown. For three animal (7.1, 9.1 and 9.2, all marked with arrows) the model overestimated the response at low dilution. Normal probability plots by dilution and treatment group are shown in Figure 12 and do not reveal departure for the normality assumption for the error. The standardized residuals plot (Figure 13) shows that the variance pattern was well captured by the power variance function which was used in the model.

FIGURE ?? - FIGURE 13 ABOUT HERE 


\section{Computational Issues}

Nonlinear mixed models can be fitted using the nlme() library in Splus or SAS procedure NLMIXED. In this section we discuss the main relevant differences between the two packages.

\subsection{Fitting Subject-Specific Models}

In SAS, Model 1 can be fitted using the PROC NLMIXED procedure. Adding a simple BY statement in the procedure allows fitting the model for each rat individually.

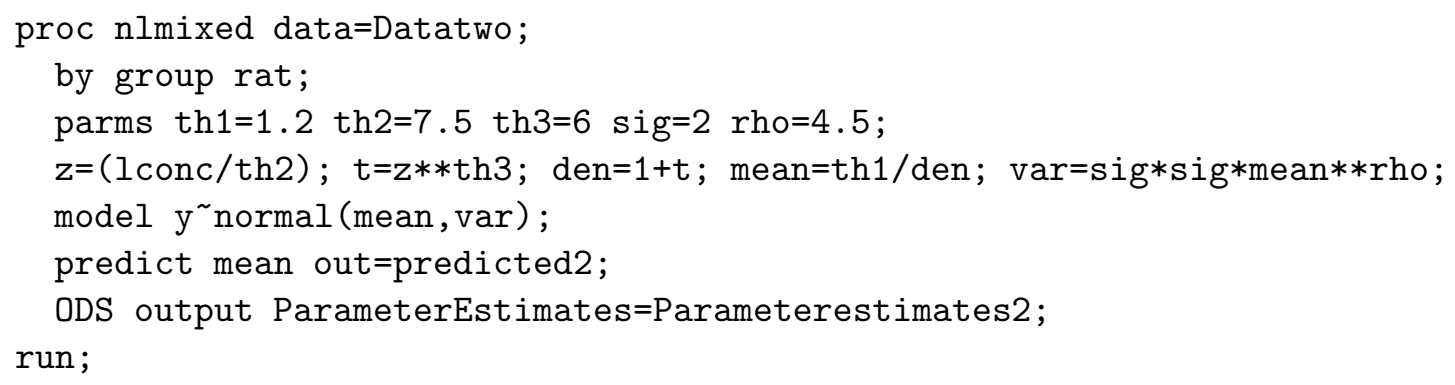

Using Splus, subject-specific model can be easily obtained using nlsList()

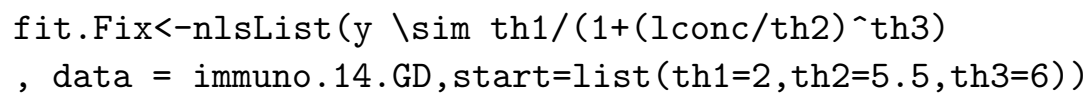

The advantage of SAS is that procedure NLMIXED allows to adjust the residual error structure while this cannot be done in nlsList which only allows a constant variance. This can be overcome by using the function gnls() in SPlus. However using gnls() for individuals models requires to program a loop which fits an individual model at each step. If one of the animals does not give a fit (which is the case for a few rats in this dataset) the loop will not be completed. In SAS the procedure is not stopped but results, in the worse case, in missing values for the individual parameters for such rat.

\subsection{Mixed Effects Approach}

The mixed effect model (10) can be fitted SAS procedure NLMIXED in the following way

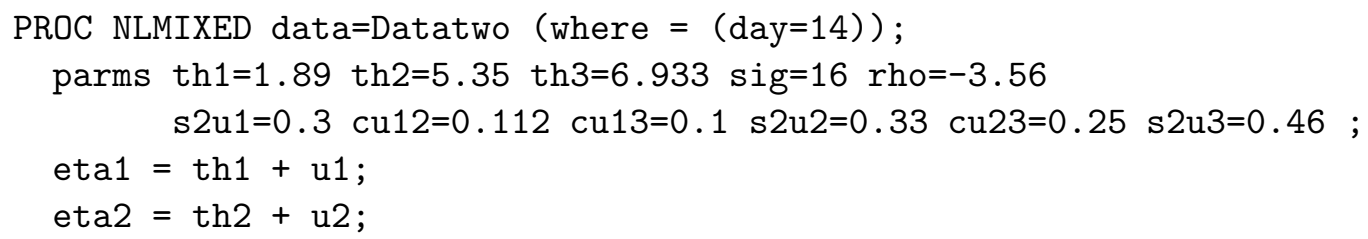




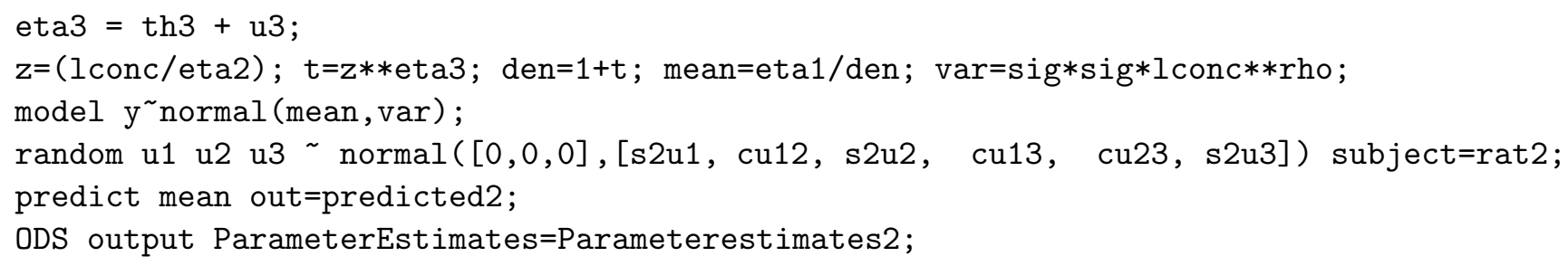

Fitting the same model in Splus requires to use the nlme() function.

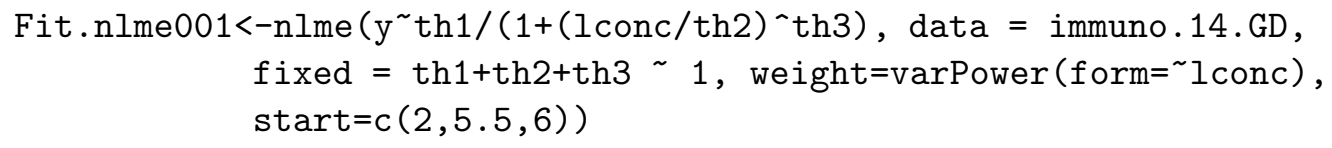

The nlme library allows an easy choice between different structures for the random effects covariance matrix (D matrix) while this is not possible in SAS. In addition, the graphical options related to the nlme() function allow quick and easy production of a different range of important graphs. The same graphs can be produced in SAS but need considerably more data manipulation and programming.

\section{Figure1:}

immuno.14.GD<-groupedData ( $\mathrm{y}^{\sim}$ lconc|rat, data=immuno.14)

plot (immuno.14.GD, outer= ${ }^{\sim}$ group, $y \lim =c(0,4)$ )

Figure2:

immuno.14.GD2<-groupedData ( $\mathrm{y}^{\sim}$ conc|rat, data=immuno.14)

plot (immuno.14.GD, outer $=^{\sim}$ group, ylim=c $(0,4)$ )

Figure3:

fit.Fix<-nlsList (y ModFun(lconc, th1, th2, th3), data = immuno.14.GD, start $=$ list $(\operatorname{th} 1=2, \operatorname{th} 2=5.5, \operatorname{th} 3=6))$

plot (augPred (fit.Fix), layout $=c(3,5)$, aspect $=0.5$, col $=1, p c h=" * "$ )

Figure5:

plot (intervals (fit.Fix), layout $=c(3,1), \operatorname{cex}=1.5)$

Figure6:

Fit.mixed1<- nlme(fit.Fix)

plot (Fit.mixed1, resid (. , type="p") lconc|group, abline=0)

Figure8:

Fit.mixed3<-update (Fit.mixed1, weight=varPower $($ form= 1 conc $)$ )

pairs (Fit.mixed3) 
Figue 10-11:

xyplot (immuno.14.GD\$y Fit.mixed3c\$fitted [, 2] | immuno.14.GD $\$$ rat, layout $=c(5,3)$, panel $=$ function $(x, y)\{$

panel.grid()

panel.xyplot $(\mathrm{x}, \mathrm{y}, \mathrm{type}=" \mathrm{p} ", \mathrm{pch}=" * ", \mathrm{col}=1)$

panel.abline $(0,1)$

\},

$\mathrm{xlab}=$ "Fitted values", $\mathrm{ylab}=$ "y", aspect=1)

Figure13:

plot (Fit.mixed3c, resid (., type="p") lconcl group, abline=0)

Table 5 presents the parameter estimates for a model which was fitted in SAS and Splus.

TABLE 5 ABOUT HERE

It can be seen that the results are almost identical. Only minor differences between the parameter estimates as well as the estimated SE exist. The reason for these differences can be explained by the default methods used by the two softwares to approach the likelihood and find the optimum conditions. In SAS the Adaptive Gaussian Quadrature (Pinheiro and Bates 1995) is used to approximate the likelihood in combination with the dual Quasi Newton optimization algorithm (Thisted 1988). Splus carries out the likelihood approximation using the LME proposed by Lindstrom and Bates (Lindstrom and Bates 1990) in combination with the Gauss-Newton optimization method.

\section{Discussion}




\section{References}

Bates, D. and Pinheiro, J. (2000) Mixed effects models in S and Splus, Springer.

European Agency for the Evaluation of Medicinal Products (EMEA), Committee for Proprietary Medicinal Products (CPMP). Note for Guidance on Repeated Dose Toxicity (CPMP/SWP/1042/99). Adopted July 2000.

Japanese Ministry for Health, Labour and Welfare (MHLW) and Japanese Pharmaceutical Manufacturing Association (JPMA). Draft guidance. December 2001

Lindsay, J.K. (2001) Nonlinear models in medical statistics, Oxford university press.

Vonesh, E.F. and Chinchilli, V.M. (1997) Linear and nonlinear model for the analysis of repeated measurements, Marcel Dekker.

US Food and Drug Administration (FDA), Center of Drug Evaluation and Research (CDER). Immunotoxicology Evaluation of Investigational New Drugs. Adopted October 2002. 
Table 1: Univariate and multivariate ANOVA.

\begin{tabular}{l|l}
\hline Parameter & F (P-value $)$ \\
\hline$\theta_{1}$ & $1.18 \times 10^{-7}(0.999)$ \\
$\theta_{3}$ & $0.0976(0.757)$ \\
$\theta_{3}$ & $0.251(0.620)$ \\
\hline multivariate & $0.986(0.947)$ \\
\hline
\end{tabular}

Table 2: Parameter estimates for the fixed effects.

\begin{tabular}{l|l}
\hline Parameter & Two stage model \\
\hline$\beta_{1}$ & $1.791(0.155)$ \\
$\beta_{2}$ & $5.496(0.205)$ \\
$\beta_{3}$ & $8.156(0.535)$ \\
$\gamma_{1}$ & $0.000075(0.219)$ \\
$\gamma_{2}$ & $-0.091(0.291)$ \\
$\gamma_{3}$ & $-0.379(0.756)$ \\
\hline
\end{tabular}

Table 3: Parameter estimates for the fixed effects and the variance components

\begin{tabular}{l|l|l|l|l}
\hline Parameter & Model 1 & Model 2 & Model 3 & Model 4 \\
$\operatorname{Cov}(\boldsymbol{b})$ & $\mathbf{D}_{1}$ & $\mathbf{D}_{1}$ & $\mathbf{D}_{2}$ & $\mathbf{D}_{2}$ \\
$\operatorname{Var}(\boldsymbol{\varepsilon})$ & $\sigma^{2}$ & $\sigma^{2}\left|C_{i j}\right|^{2 \delta}$ & $\sigma^{2}\left|C_{i j}\right|^{2 \delta}$ & $\sigma^{2}\left|C_{i j}\right|^{2 \delta}$ \\
\hline$\beta_{1}$ & $1.751(0.107)$ & $1.871(0.106)$ & $1.875(0.109)$ & $1.870(0.110)$ \\
$\beta_{2}$ & $5.513(0.129)$ & $5.366(0.112)$ & $5.369(0.113)$ & $5.379(0.111)$ \\
$\beta_{3}$ & $8.002(0.397)$ & $6.944(0.141)$ & $6.953(0.142)$ & $6.970(0.134)$ \\
$\gamma_{1}$ & & & & $-0.011(0.156)$ \\
$\gamma_{2}$ & & & & $0.127(0.157)$ \\
$\gamma_{3}$ & & & 15.753 & 14.988 \\
\hline$\sigma$ & 0.125 & 16.592 & -3.531 & -3.494 \\
$\delta_{2}$ & & -3.563 & -488.673 & -483.699 \\
\hline AIC & -67.865 & -488.546 & -458.549 & -443.534 \\
BIC & -34.394 & -451.728 & $0.189)$ \\
\hline
\end{tabular}


Table 4: Parameter estimates for the fixed effects.

\begin{tabular}{l|l|l|l|l}
\hline Parameter & $\begin{array}{l}\text { Two stage model } \\
\text { without treat. effects }\end{array}$ & $\begin{array}{l}\text { mixed effects model } \\
\text { model 3 }\end{array}$ & $\begin{array}{l}\text { Two stage model } \\
\text { with treat. effects }\end{array}$ & $\begin{array}{l}\text { mixed effects model } \\
\text { model } 4\end{array}$ \\
\hline$\beta_{1}$ & $1.791(0.107)$ & $1.875(0.109)$ & $1.791(0.155)$ & $1.870(0.110)$ \\
$\beta_{2}$ & $5.450(0.143)$ & $5.369(0.113)$ & $5.496(0.205)$ & $5.379(0.111)$ \\
$\beta_{3}$ & $7.966(0.373)$ & $6.953(0.142)$ & $8.156(0.535)$ & $6.970(0.134)$ \\
$\gamma_{1}$ & & & $0.000075(0.219)$ & $-0.011(0.156)$ \\
$\gamma_{2}$ & & & $-0.091(0.291)$ & $0.127(0.157)$ \\
$\gamma_{3}$ & & $-0.379(0.756)$ & $0.228(0.189)$ \\
\hline
\end{tabular}

Table 5: Parameter estimates for the fixed effects.

\begin{tabular}{l|l|l}
\hline Parameter & SAS & SPlus \\
\hline$\hat{\beta}_{1}$ & $1.891(0.105)$ & $1.871(0.106)$ \\
$\hat{\beta}_{1}$ & $5.350(0.115)$ & $5.366(0.112)$ \\
$\hat{\beta}_{1}$ & $6.933(0.146)$ & $6.944(0.141)$ \\
\hline
\end{tabular}

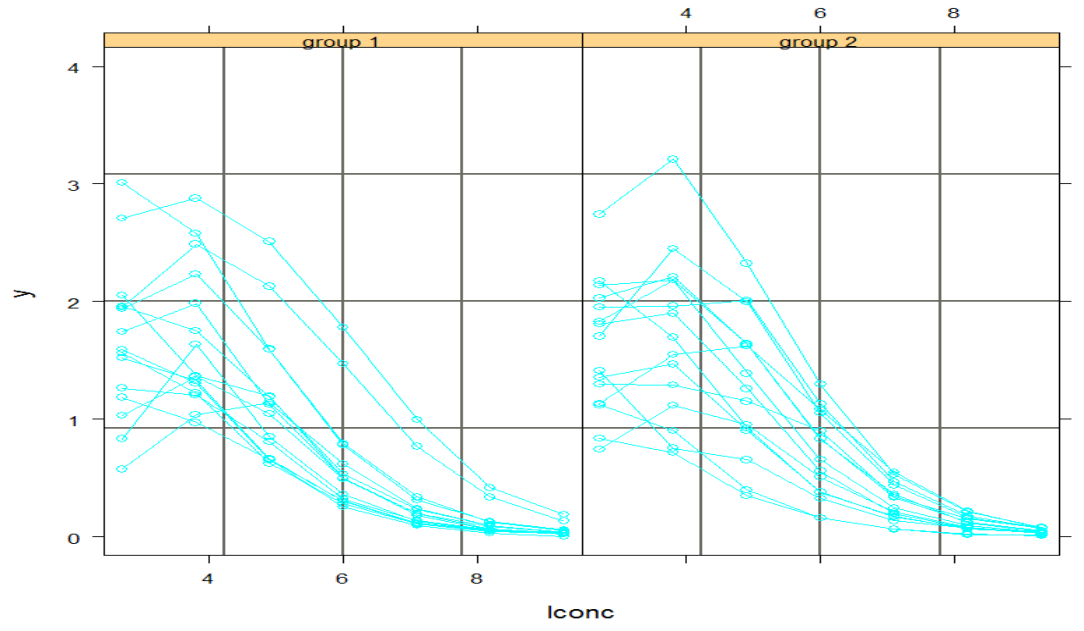

Figure 1: animals profiles at day 14 by treatment group. Response versus log dilution. 


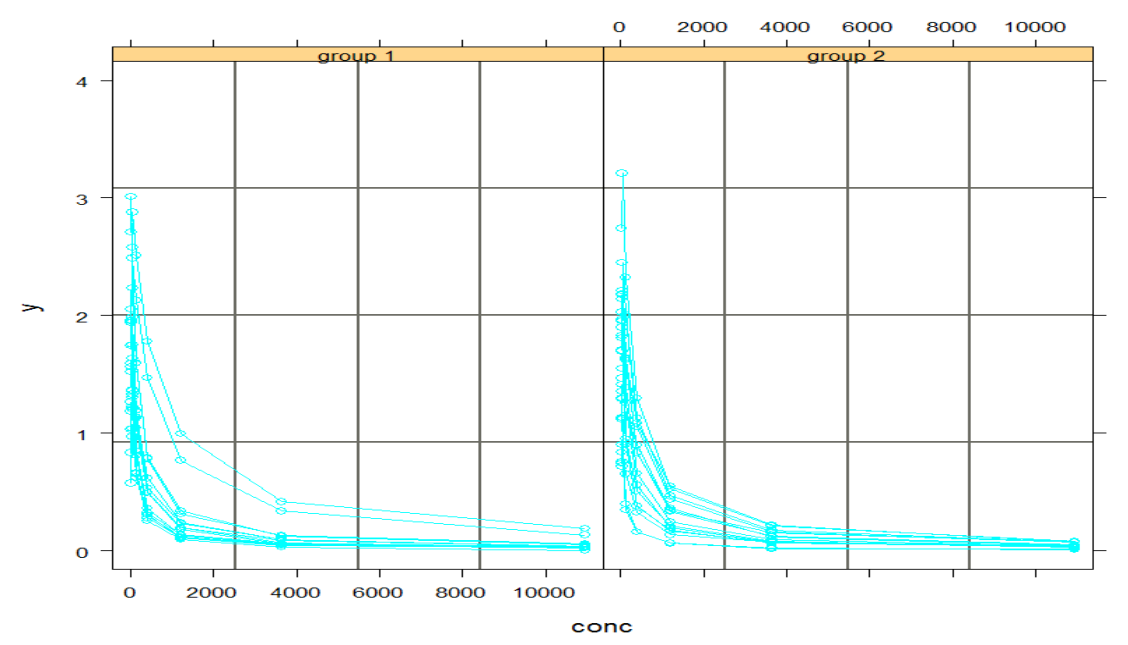

Figure 2: animals profiles at day 14 by treatment group. Response versus dilution.

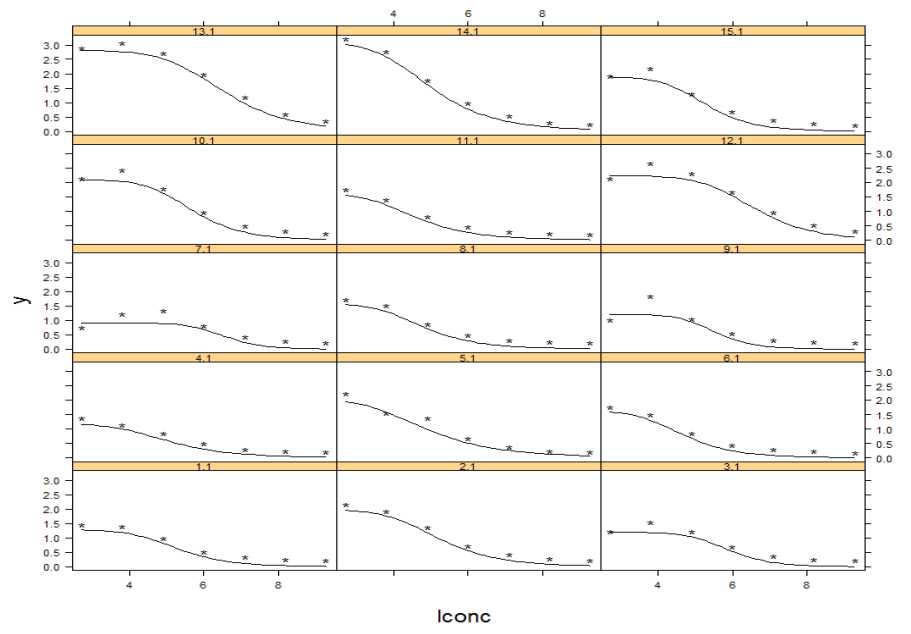

Figure 3: Observed data and animal specific models (for the first treatment group). 


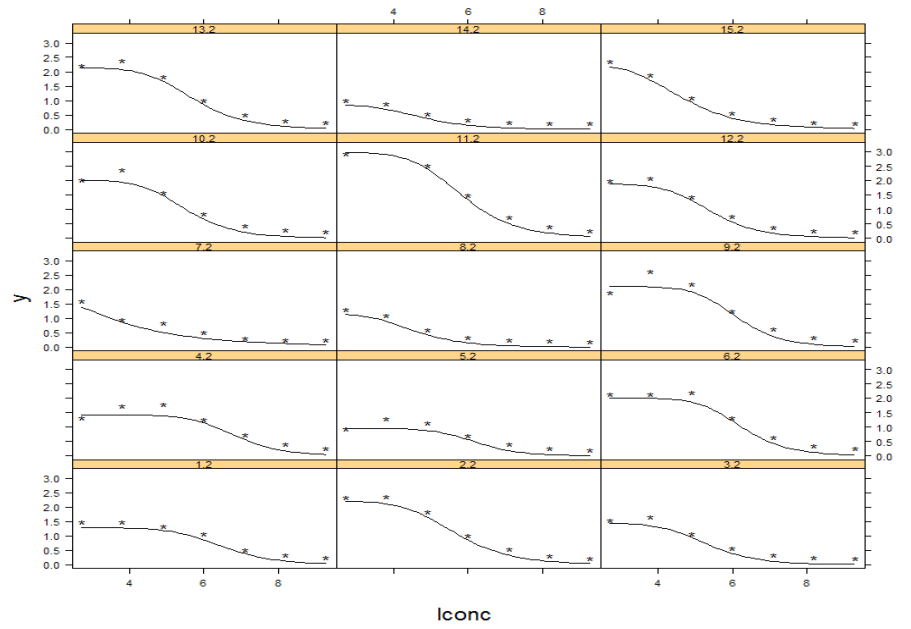

Figure 4: Observed data and animal specific models (for the second treatment group).

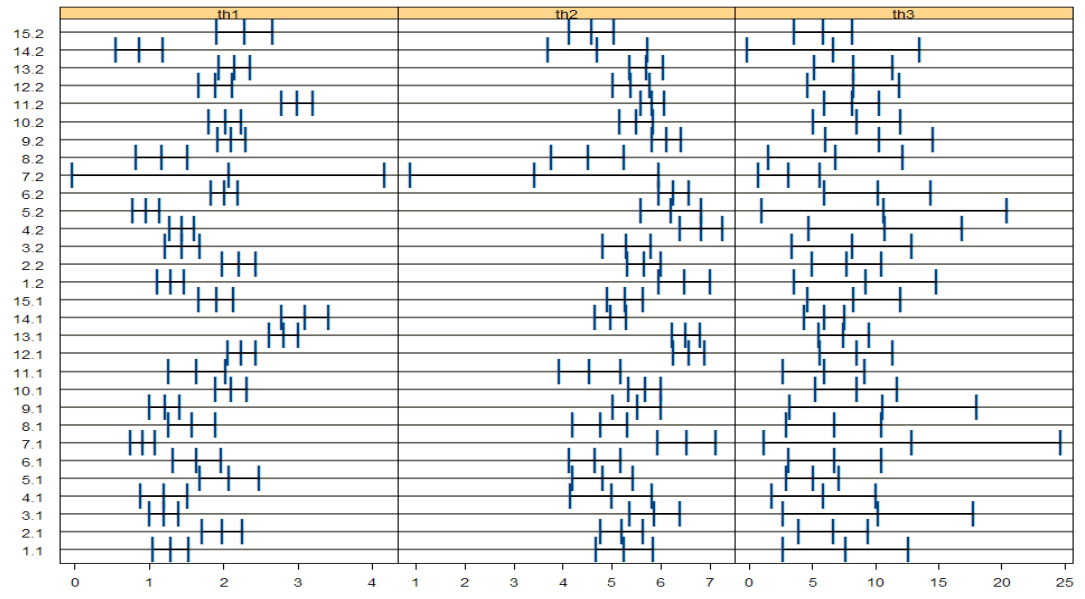

Figure 5: $M L$ estimate from $\boldsymbol{\theta}$ obtained from the individuals models. 


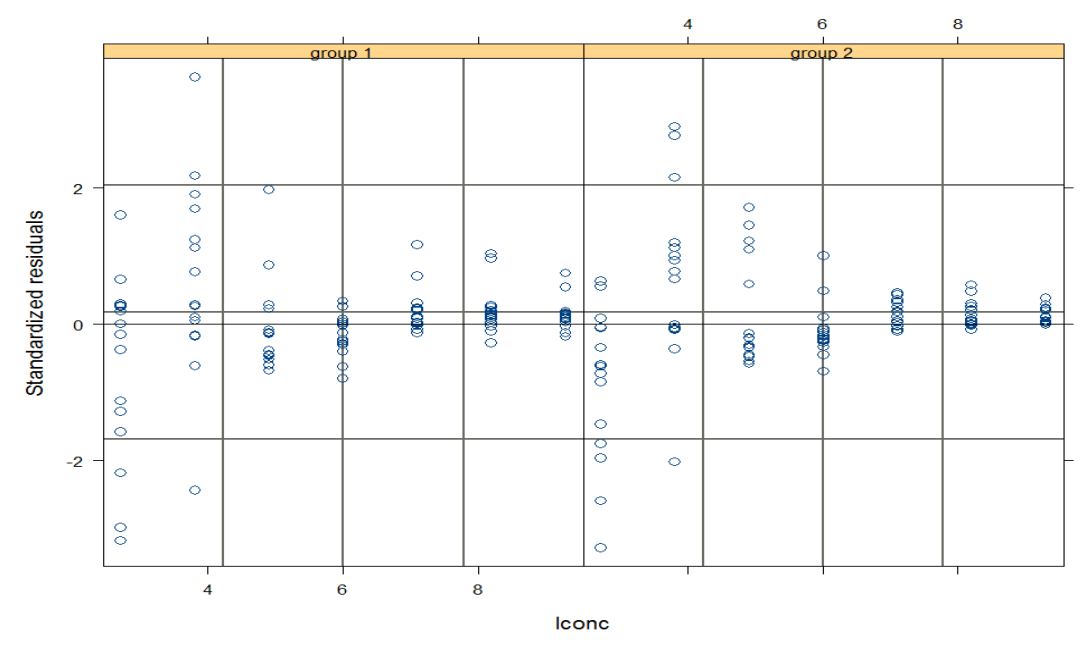

Figure 6: Standardized residuals by treatment group obtained from model 1.
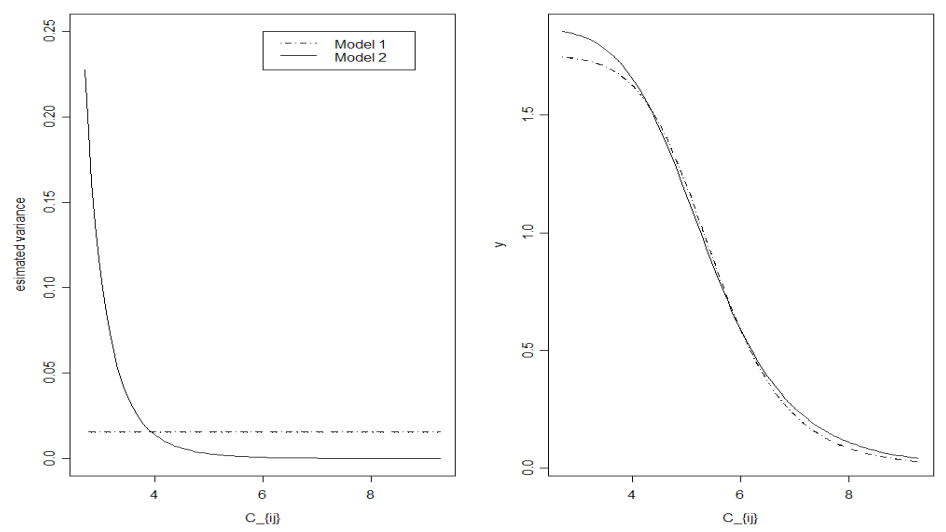

Figure 7: Left panel: variance functions for model 1 and model 2. Right panel: predicted model (population models). 


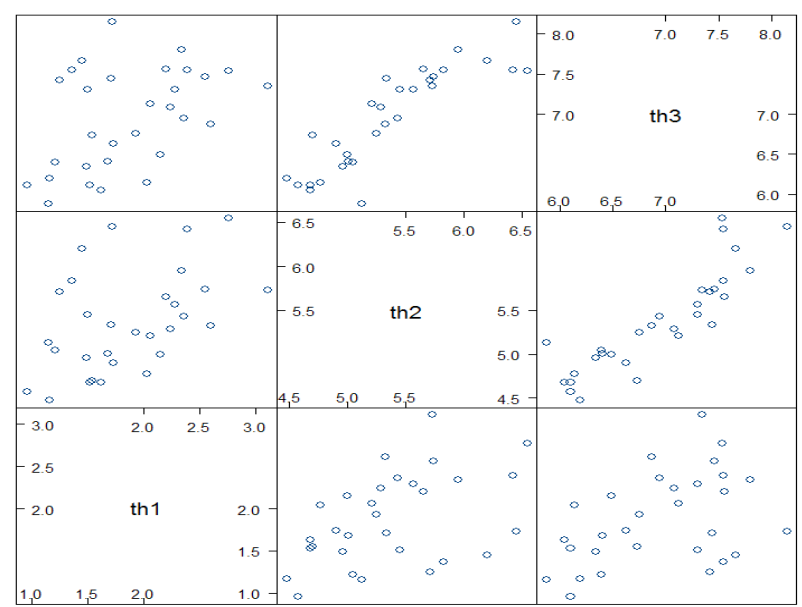

Figure 8: Scatterplot matrix for the random effects.

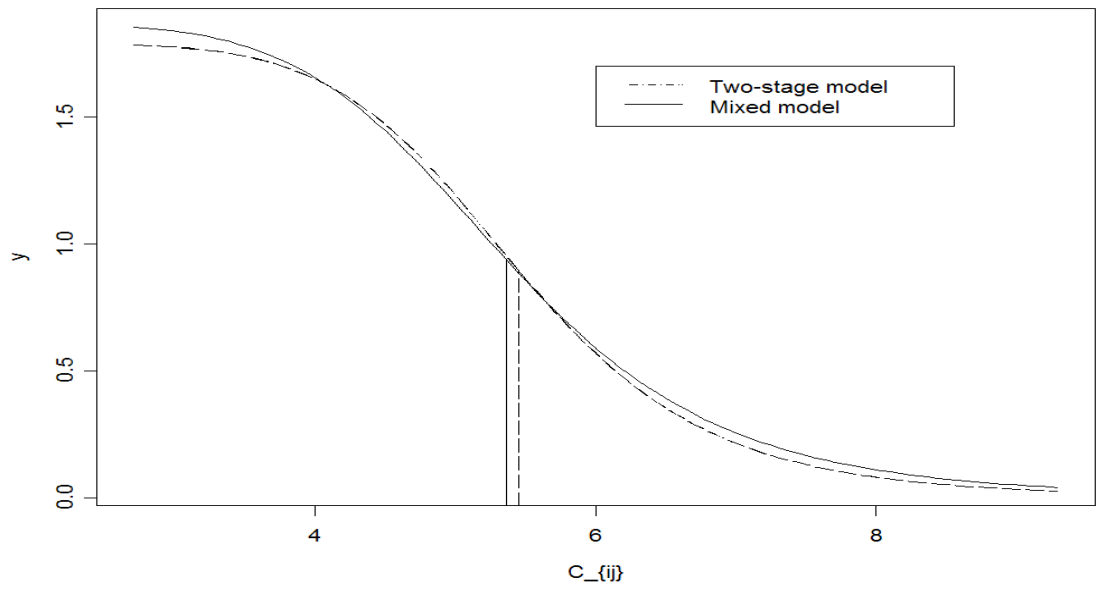

Figure 9: Population models. Model 3 and the two stage model. 


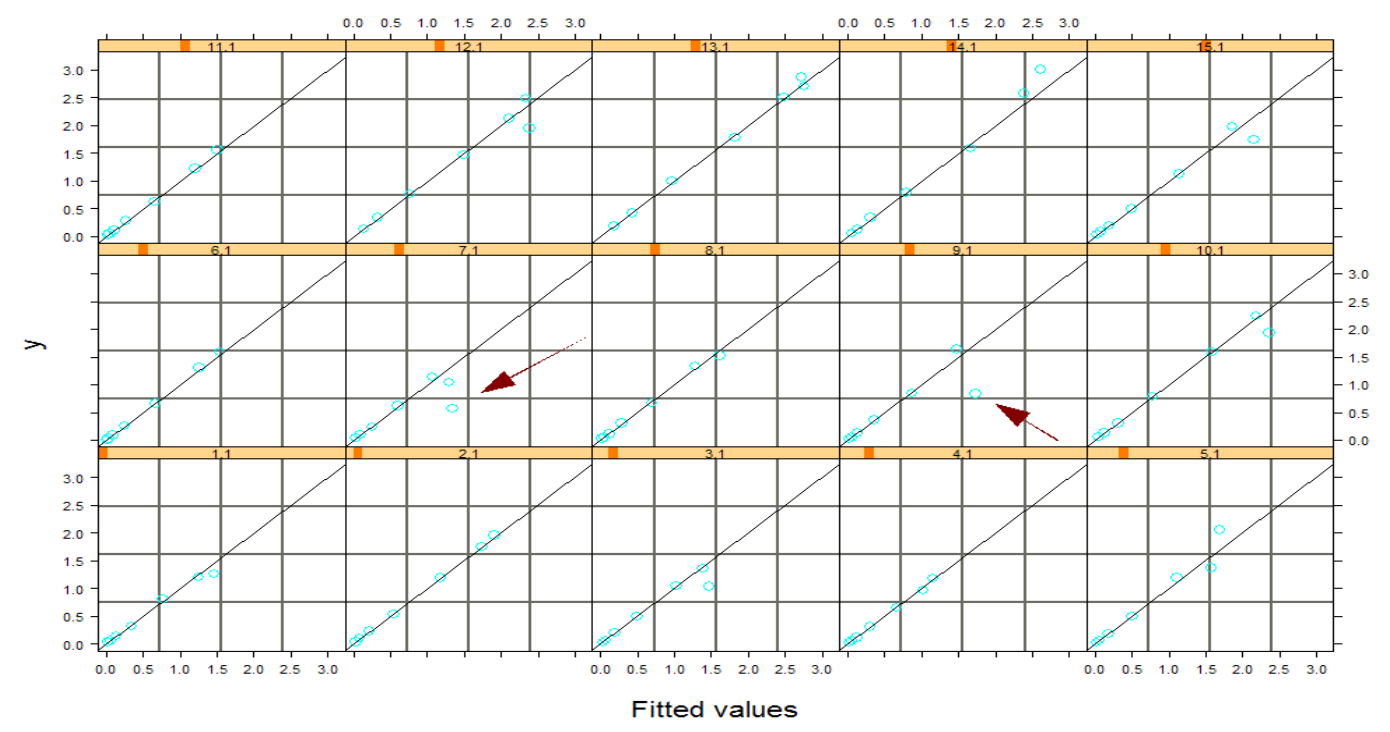

Figure 10: Model 3. Observed versus predicted values in the first treatment group.

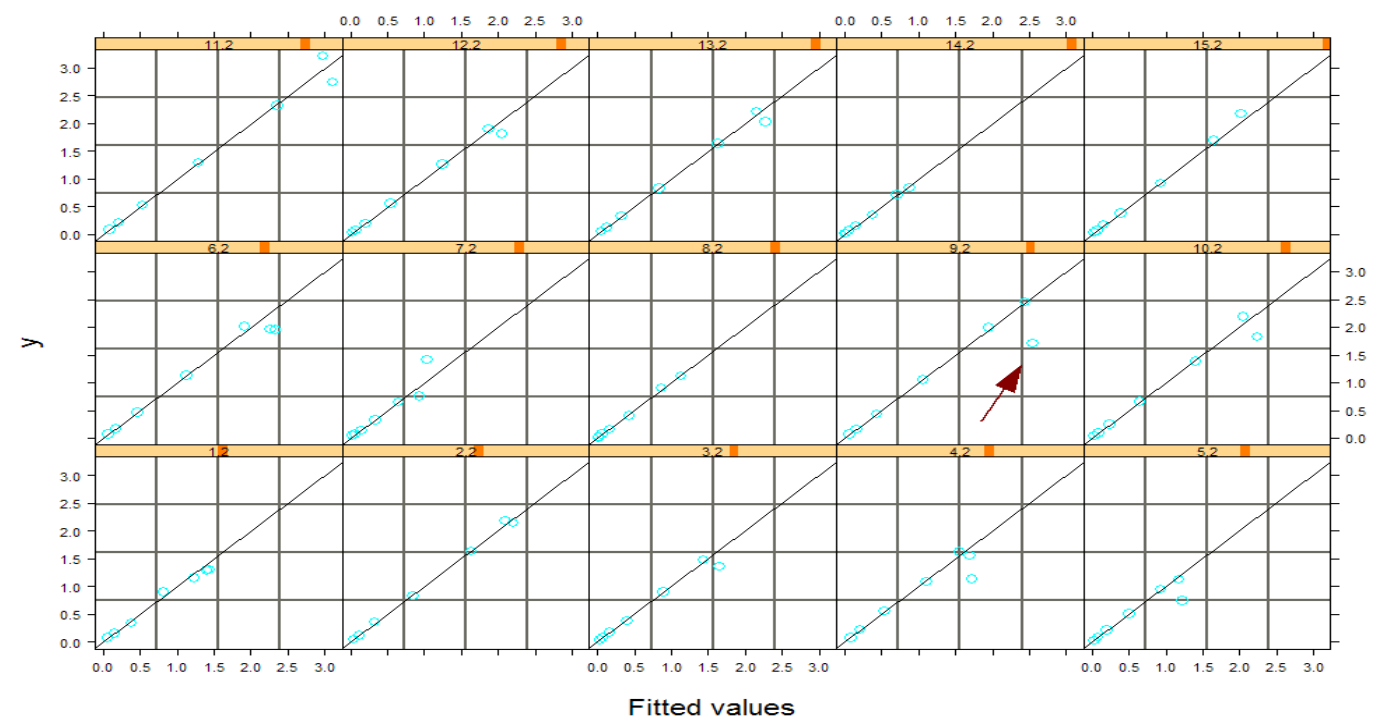

Figure 11: Model 3. Observed and predicted values for the second treatment group. 


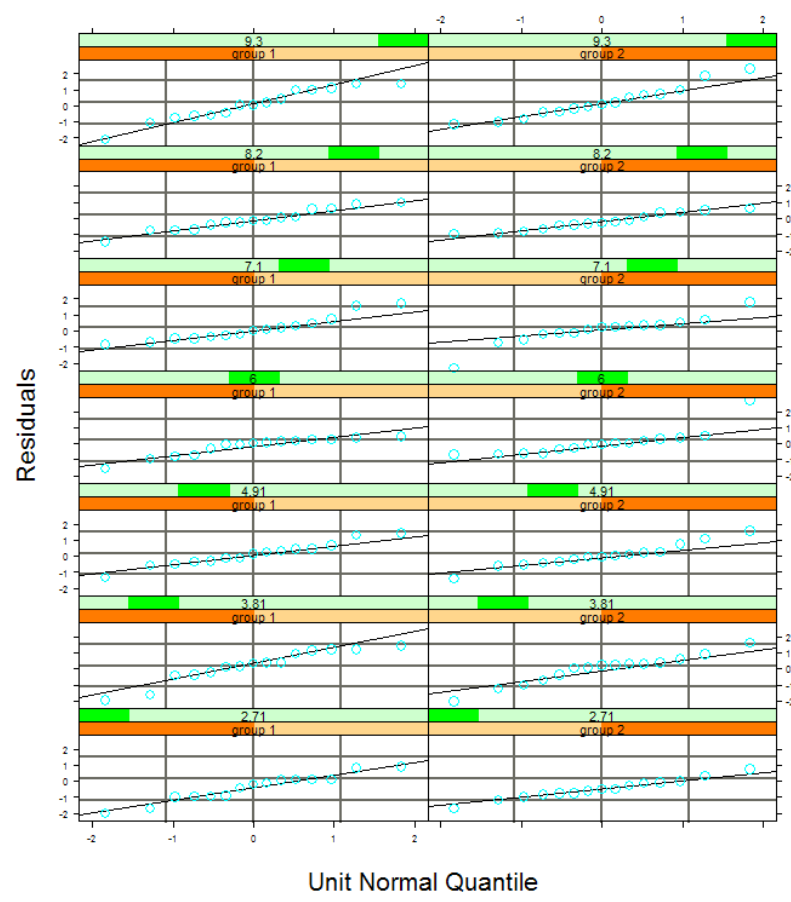

Figure 12: Model 3. Quantile-quantile normal probability plot by dose level and treatment group. The left and the right columns present the qqnormal plots for the first and the second treatment group, respectively.

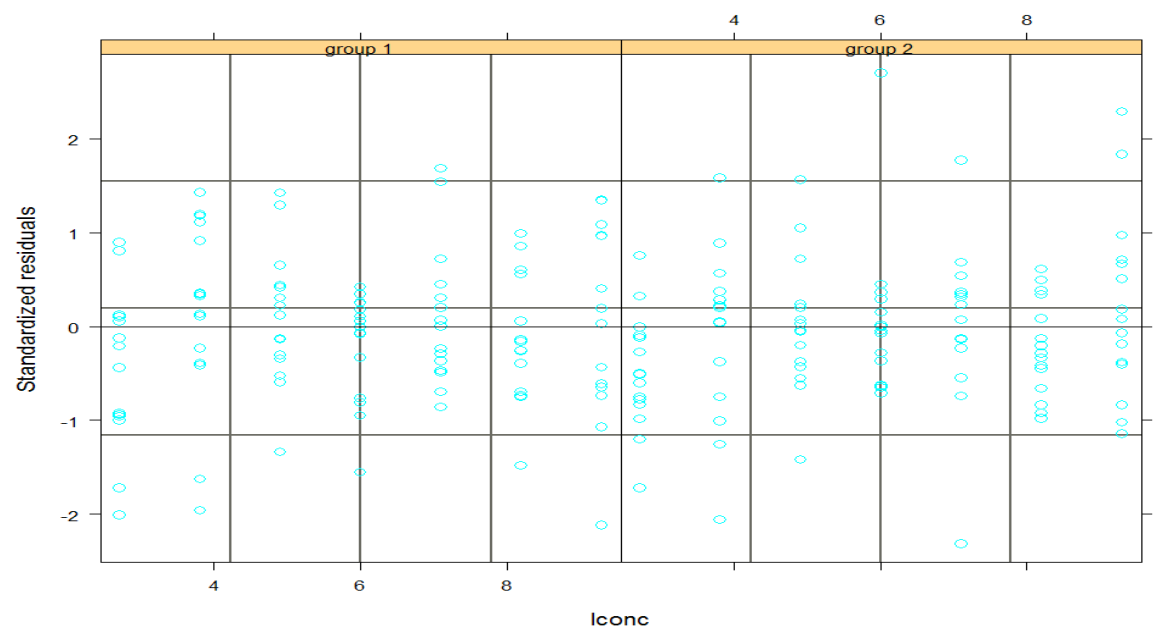

Figure 13: Model 3. Standardized residuals obtained from the final model by treatment group. 